\title{
Reliability of redundant ductile structures with uncertain system failure criteria
}

\author{
BAIDURYA BHATTACHARYA ${ }^{1, *}$, QIANG LU ${ }^{2}$ and \\ JINQUAN ZHONG ${ }^{3}$
}

${ }^{1}$ Department of Civil Engineering, Indian Institute of Technology, Kharagpur, WB 721302

${ }^{2}$ Department of Mechanical Engineering, Northwestern University, Evanston, IL 60208, USA

${ }^{3}$ Formerly graduate student, Civil and Environmental Engineering Department, University of Delaware, Newark, DE, USA

e-mail: baidurya@iitkgp.ac.in

MS received 7 May 2008; revised 2 March 2009

\begin{abstract}
Current reliability based approaches to structural design are typically element based: they commonly include uncertainties in the structural resistance, applied loads and geometric parameters, and in some cases in the idealized structural model. Nevertheless, the true measure of safety is the structural systems reliability which must consider multiple failure paths, load sharing and load redistribution after member failures, and is beyond the domain of element reliability analysis. Identification of system failure is often subjective, and a crisp definition of system failure arises naturally only in a few idealized instances equally important. We analyse the multi-girder steel highway bridge as a $k$ out of $n$ active parallel system. System failure is defined as gross inelastic deformation of the bridge deck; the subjectivity in the failure criterion is accounted for by generalizing $k$ as a random variable. Randomness in $k$ arises from a non-unique relation between number of failed girders and maximum deflection and from randomness in the definition of the failure deflection. We show how uncertain failure criteria and structural systems analyses can be decoupled. Randomness in the transverse location of trucks is considered and elastic perfectly plastic material response is assumed. The role of the system factor modifying the element-reliability based design equation to achieve a target system reliability is also demonstrated.
\end{abstract}

Keywords. System reliability; Monte Carlo simulation; importance sampling; uncertainty; bridge.

*For correspondence 


\section{Introduction}

The structural design process is a continual effort to balance performance with safety and economy. Although it is possible to merge the twin constraints of safety and economy into a single format by minimizing the total life-cycle expected cost of the structure (Frangopol et al 1997; Wen 2001), it is almost universally common to consider the requirement of safety in a separate and explicit manner. Structural safety can most rationally be addressed using principles of structural reliability which consider uncertainties and incomplete knowledge about structural loads, strength, geometry and behaviour in a probabilistic format. Accordingly, structural design codes over the past three decades or so have begun to move away from a deterministic safety factor based approach to a reliability-based one: at the current state of the art, most of these codes make use of design equations that involve partial safety factors calibrated to achieve a target reliability (on the average) and intended to reflect the relative uncertainties in the load and resistance parameters. Most of these design approaches also share a common feature that the design philosophy is component-based (Galambos 1990; Moses 1997). The safety check is performed on a component basis with the implicit expectation that the structural system will be safe as long as all its component reliabilities are satisfactory.

Although this process has served the profession well, component-based design makes it difficult to achieve uniform reliability in all structural systems designed by any particular specification. After all, it is the failure of the system that is of ultimate significance to the engineer and the public, hence, in addition to the components having adequate reliability, it is paramount that the system reliability meets its target value-a target that is commensurate with the consequences of system failure.

Further, the definition of what constitutes system failure is not always a straightforward matter and is beset with subjectivity for real structures. Failure criteria in terms of excessive deflection or incipient instability are commonly accepted: but the precise numerical definitions or functional forms of these limit states are often chosen arbitrarily. The fact that structural analyses for 'failure' identification should involve material and/or geometric nonlinearities does not help matters either. Well-defined criteria of system failure are possible only in a few idealized cases. Yet, the all-too-common uncertainty surrounding system failure limit states has not received adequate attention in the vast body of structural systems reliability literature available today.

A well-designed structural system should, first of all, have adequate safety margin in intact condition; it should also be sufficiently indifferent to the failure of at least the first few members. Such desirable behaviour arises from structural indeterminacy coupled with excess capacity of the critical members (commonly known as redundancy), load redistribution, nonbrittle member failure, etc. An instructive example is provided in Liu \& Moses (1991) where a girder bridge, because of 'over-optimization' in member sizing, loses all structural redundancy and ends up with a system reliability equal to that of the critical girder. A structural system may also possess system failure modes such as excessive global deformation that do not necessarily involve any component failure. Further, there is no assurance that the component-based safety criteria will be adequate if they are applied in relatively novel structures where there may be little experience and unexpected failure modes such as a progressive collapse sequence (Bhattacharya et al 2001). It is thus clear that the system reliability of a structure may not possess a high correlation with the reliability of its critical components and a design by components cannot guarantee an optimally designed system at least from reliability considerations. 
Nevertheless, it is possible, in some rudimentary but practically appealing way, to modify the component design equation that takes into account systems effects (e.g. see Moses 1982; Galambos 1990; AASHTO 1994) and ensure the target system reliability for the given structural configuration and loading. This technique of course would require a clear understanding of the system failure criteria, load sharing and load redistribution after initial component failures, post-failure material behaviour, and possible dependence among the basic variables.

It is in the above context that a systems analysis on the reliability of steel girder highway bridges is conducted in this paper. Particular emphasis is placed on how uncertainty in failure criteria can be incorporated in the analysis. Statistical dependence among (i) girder strengths and (ii) truck loads in case of multiple presence is also considered. A 'system factor' representing the systems effects mentioned above to be used in an LRFD type component design equation is developed such that the target system reliability is achieved. No aging effect on structural strength or accidental damage to members is considered.

\section{Background}

\subsection{Analysis of structural reliability}

Significant uncertainties, reducible or intrinsic, may be associated with some or all of the parameters (e.g. the geometric, material, loading, etc.) that govern the performance of a structure. It is most rational to treat such parameters, denoted as basic variables of the problem, as random variables or random processes/fields depending on whether they are temporally/spatially invariant or not. Structural reliability, then, is defined as the probability that the entire spatial domain of the structure will perform satisfactorily during a specified service life under given operating conditions.

If it is possible to define a single differentiable performance function $g(\mathbf{X})$ of the basic variables $\mathbf{X}$ such that $g>0$ implies satisfactory performance (or a safe state) and $g<0$ implies failure of the structure, then we have what is known as an element reliability problem at hand. The limit state surface for the structure, which separates the safe state from the failed state, is given by $g=0$. An element reliability problem is most naturally realized in the case of a single structural component (or, more generally, a critical cross-section of a component) in a single failure mode (such as tensile); in such cases the function $g$ is derived from analytical modelling. Methods of varying elegance and sophistication, most notable of which is FORM (first order reliability method), are available to solve component reliability problems.

The difference between an element and a system in a reliability analysis context is somewhat arbitrary and largely dependent on the scale of interest. Indeed, a problem of tensile failure of a prismatic rod made of a brittle material that can be treated as a simple component reliability problem from a continuum viewpoint may amount to an intractable network reliability problem from micro-structural considerations. For practical purposes, it is mostly the availability of a single, differentiable and closed-form performance function that separates an element reliability problem from a systems one.

It would be highly desirable, then, to somehow cast the performance of a structural system in terms on a single limit state (perhaps using approximate numerical techniques such as a response function fit) and thereby take advantage of the speed, elegance and accuracy of element reliability solution techniques; such formulation unfortunately remains elusive more often than not. Needless to add that structural system failure events are thankfully so rare 
(and in any case, structural systems can hardly be deemed to constitute a nominally identical sample) that the other alternative - a frequentist interpretation of structural system reliability is not feasible. The usual systems reliability formulation therefore is presented in terms of Boolean combinations of element limit states depending on the logical construct of the system in terms of its components and the definition of failure at the systems level.

The binary nature of components (being either in failed or safe states), though not a necessity, facilitates the use of standard methods such as fault or event trees (or a combination of the two), including their variants to suit the peculiarities of structural systems, to describe system failure in terms of component failure events, and hence to identify minimal cut and/or minimal path sets of the system. System reliability computation for structures is not straightforward since the component failures are not mutually independent events on account of (i) active redundancy in the structure leading to load sharing, (ii) load path dependence in case of successively applied multiple yet sustained loads, (iii) load redistribution after initial member failures for redundant structures, (iv) non-linear behaviour and non-brittle failure of the components, (v) failure sequences of different probabilities for the same cut set in a progressive collapse or incremental loading situation, and (vi) possible statistical dependence among the basic variables.

Several techniques, each tailored to address some of the above issues, have been developed over the years for tackling structural systems reliability problems. FORM, which maps the limit state equation from the basic variable space to the rotationally symmetric uncorrelated standard normal space (using methods of various sophistication including those such as Rosenblatt transformation that preserve the dependence structure), can be easily extended to simple system reliability problems. If the problem can be cast as an intersection of $m$ events (i.e. a classical parallel system), then the first order approximation to the system failure probability boils down to the evaluation of an $m$-dimensional Normal probability integration:

$$
\text { Parellel system: } P_{f, s y s}=P\left[\bigcap_{i=1}^{m} g_{i} \leq 0\right] \approx \Phi_{m}(-\boldsymbol{\beta} ; \mathbf{R}) .
$$

Where $g_{i}$ 's are the limit state surfaces in the original basic variable space $(\mathbf{X}) . \boldsymbol{\beta}$ is the vector of minimum distances from the origin to the respective limit state surfaces, $\tilde{g}_{i}$, once they have been mapped onto the uncorrelated standard Normal space $(\mathbf{u})$. $\mathbf{R}$ is the correlation matrix of the limit state surfaces linearized at the respective minimum distance points. Equation (1) becomes exact if the transformed limit state functions $\tilde{g}_{i}$ are linear in $\mathbf{u}$ which occurs if the basic variables are Normal and the original limit state functions $g_{x}(\mathbf{X})$ 's are linear in the basic variables.

For a series system type configuration, the multidimensional Normal integration approach can still be used by looking at the complementary events and using the symmetry property of the standard Normal space:

$$
\text { Series system: } P_{f, s y s}=P\left[\bigcup_{i=1}^{m} g_{i} \leq 0\right] \approx 1-\Phi_{m}(\boldsymbol{\beta} ; \mathbf{R}) \text {. }
$$

As for the evaluation of the multidimensional Normal integration in (1) and (2), it is certainly not a trivial task, and approximations have been developed over the years (Hohenbichler \& Rackwitz 1983; Breitung 1984; Tang \& Melchers 1987; Tong 1990).

For systems more general than the simple series and parallel organizations, the greatest challenge is to identify the minimal cut sets (at least the dominant ones), particularly in 
light of the circumstances peculiar to structural systems mentioned above. If the cut sets $C_{i}, i=1, \ldots, n_{c}$ can be identified for the system, (2) can be generalized as:

$$
P_{f, \mathrm{sys}}=P\left[\bigcup_{i=1}^{n_{c}} C_{i}\right]=P\left[\bigcup_{i=1}^{n_{c}}\left\{\bigcap_{j=1}^{n_{i}} g_{i j} \leq 0\right\}\right]
$$

where $g_{i j}$ is the $j^{\text {th }}$ limit state in cut set $i$. Exact solution of (3) may be impossible, thus bounds on system reliability, based on marginal events (Cornell 1967), pairs of joint events (Ditlevsen 1979) or triplets of joint events (Hohenbichler \& Rackwitz 1983) are available. Cut sets, without regard to an ordering of element failure events, are possible to be determined for elastic-perfectly plastic structures.

An ordered sequence of failure events from a cut set is variously termed in structural systems reliability analyses, sometimes with subtle differences among them, as failure sequence or failure path. To be specific, a failure sequence under incremental loading accounts for load redistribution after each component failure while a failure path does not, and leads to different events whenever load redistribution occurs after each successive component failure (Bjerager et al 1987). The terms failure mode and collapse mode, unfortunately, have been used in the literature to denote a cut set both with and without regard to ordering of failure events and have led to confusion in some cases. In this paper, "collapse mode' will imply a cut set without reference to failure order, and 'failure sequence' will imply an ordered sequence from a cut set. A path set is sometimes referred to as a stable configuration (Bennet \& Ang 1986) although this approach is rarely taken in structural problems.

For a structure with $n$ binary components, $2^{n}$ mutually exclusive system states are possible: only some of these produce system failure. If the order in which these components fail are important and need to be enumerated (either due to modelling convenience or if the physical process of failure is indeed such), the total number of distinct sequences increases to $\sum_{r=1}^{n} n ! /(n-r) !(r$ being the number of failed components) which can be a very large number even for moderately sized structures. Nevertheless, only some of these sequences are failure sequences i.e. lead to system failure in the fashion of a failure tree, and furthermore, only a subset of these failure sequences usually are dominant, i.e. have a non-negligible probability of occurrence. It is thus clear that efficient methods of identifying the dominant failure sequences (or dominant collapse modes if sequence effects are not important) are necessary for all but the simplest structures.

Depending on the structural complexity and desired accuracy of the solution, the dominant failure sequences (or collapse modes) can be found in a variety of ways: some of these involve only a deterministic analysis of the structural system while some employ a fully probabilistic analysis, and still others that use some limited probabilistic information. The assumption of rigid perfectly plastic material behaviour is fairly popular in structural system reliability analysis as it eliminates load history dependence. It is well-known that deterministic plastic mechanism analysis can lead to collapse mode identification in case of rigid-plastic framed structures, although the number of modes generated quickly becomes huge (Watwood 1979; Gorman 1981). Such deterministic rules have been variously adapted to search for the probabilistically dominant collapse modes by (i) creating linear combinations of those basic mechanisms that have the lowest reliability indices (the beta-unzipping method-Thoft-Christensen \& Murotsu 1986), (ii) using linear programming (Corotis \& Nafday 1989), (iii) stochastic programming (Zimmerman et al 1993), (iv) genetic algorithms (Shao \& Murotsu 1999). The probabilistically dominant failure sequences can be searched using truncated enumeration schemes that 
include the branch and bound method (Thoft-Christensen \& Murotsu 1986) and, importantly, the incremental loading method which we discuss next.

Incremental loading of a structure in which the magnitude of the vector of external load variables is slowly increased from zero up to a pre-determined cut-off condition (while keeping the direction of the vector constant) and in which the structural state is updated within the confines of static equilibrium at each successive component failure is a versatile method for identifying failure sequences (Karamchandani 1987; Moses 1997). Some or all the basic variables in such incremental analysis may be set at their mean values; alternately, random samples of the basic variables may be generated repeatedly until the dominant sequences have been determined. The incremental loading method is particularly useful (and often the only way out) when component failure is multistate instead of the usual binary (Karamchandani \& Cornell 1992b), material behaviour is brittle, semi-brittle or non-linear instead of ideal plastic (Karamchandani \& Cornell 1992a), and system failure occurs not due to formation of a mechanism, but due to excessive deformation or a specified drop in structural stiffness with regard to specified degrees of freedom. Nevertheless, one potential drawback of the incremental analysis method is its quasi-static assumption of structural behaviour: the load duration needs to be sufficiently long to allow potential redistribution of load effects throughout the system.

\subsection{Reliability of Bridges}

In the allowable stress method of bridge design (also termed as working stress design or WSD), the uncertainties in loads and element resistance are taken into account using a single factor of safety. Safety checks in WSD is by definition performed in the elastic domain with the implicit assumption that the structure thus designed would perform adequately in the inelastic domain near failure. In 1994, as a result of NCHRP Project 12-33, the reliability-based AASHTO Load and Resistance Factor Design (LRFD) Highway Bridge Design Specifications were published (AASHTO 1994). The load and resistance factors (LRFs) were calibrated based on a global population of bridges (NCHRP 1999). The benefit of LRFD over WSD is that safety checks can be performed beyond yield close to collapse conditions, and designs using LRFD have a more uniform level of safety across a range of configurations.

Nevertheless, as stated previously, AASHTO LRFD specifications are essentially an element-based approach to ensuring structural reliability although the importance of system redundancy is also recognized in a qualitative way in the AASHTO code.

The need to estimate system reliability of bridge structures has long been recognized. Liu \& Moses (1991) considered reliability of girder bridges that have sustained damage to one or more girders. This damage could arise from accidents (fire, collision, etc.) or loading/environment (corrosion, fatigue, etc.). Estes \& Frangopol (1991) developed a lifetime repair strategy for bridges that minimized total lifetime repair costs while maintaining a minimum system reliability. Time-dependent structural damage as well as increase of live loads over time were considered. Moses (1997) proposed a general method of optimization based on AASHTO LRFD code considering system reliability effects. A comprehensive analysis of redundancy of bridge superstructures was performed in NCHRP Project 12-36 (Ghosn \& Moses 1998). Bridge system reliability was considered intact as well as damaged conditions. Both ultimate and functionality limit states were considered in the intact condition, while the only concern in damaged condition was the ultimate limit state. The ultimate system limit state was defined as either formation of a mechanism leading to collapse, or extensive damage such as crushing of concrete in main members. The functionality limit state corresponded to excessive permanent deformation in the main members caused by live load; the limit chosen was $1 / 100^{\text {th }}$ of the span length and was based on 'dangerously high levels' of deformation of 
the bridge deck perceived by bridge owners and users. Three nominal system reserve ratios, $R_{u}, R_{f}$ and $R_{d}$ corresponding to ultimate, functionality and damaged condition limit states, respectively, were computed based on nominal load and resistance parameters, using nonlinear finite element analyses. Minimum acceptable values of $R_{u}, R_{f}$ and $R_{d}$ were determined by calibrating the excess system reliability indices (relative to the reliability index of the first member that fails) to existing structures that were known to be sufficiently redundant. Finally, 'system factors' multiplying the element strength in a component level design equation were provided for a number of common bridge types and geometries.

In the following sections, the system reliability formulation of a typical steel girder bridge as an $r$-out-of- $n$ active parallel system is described. Practical difficulties in establishing system failure criteria are highlighted and related uncertainties are incorporated in the reliability formulation. System reliability of the said bridge as a function of (i) dependence among basic variables, and (ii) the system factor used in element design, is illustrated.

\section{Girder bridge as a random $r$-out-of- $n$ active parallel system}

A structure must perform satisfactorily in all relevant load cases (e.g. live load, wind, earthquake, etc.) during its service life. The overall system failure event thus is the union:

$$
F_{\mathrm{sys}}=\bigcup_{j=1}^{n_{L}} F_{\mathrm{sys}}^{(j)}=\bigcup_{j=1}^{n_{L}} \bigcup_{i=1}^{n_{j}} C_{i}^{(j)},
$$

where the total number of load cases is $n_{L}$ and $F_{\text {sys }}^{(j)}$ is the system failure in the $j^{\text {th }}$ load case. In general, each load case is defined by its own vector of basic variables, $\mathbf{X}^{(j)}$, and has its own set of minimal cut sets $C_{i}^{(j)}$ (depending on how failure is defined in a given load case) that are functions of $\mathbf{X}^{(j)}$. It is clear that, for the various $j$ 's, the $\mathbf{X}^{(j)}$ 's may share strength, geometry as well as load random variables.

Subjectivity often exists in system failure criteria for structural systems and requires application of engineering judgment in most cases. Determining which sets of events constitute failure of a structural system, short of a complete collapse, depends as much on the perceptions of owners/users as on the technical aspects. A brief summary showing the range of criteria/definitions used for girder bridge failure is presented in the following.

\subsection{A summary of existing failure criteria}

Cho \& Ang (1989) used the stable configuration approach; in an example involving system reliability of a five girder reinforced concrete $T$ beam bridge, they defined the dominant stable configuration as either no overstressed girder or one overstressed beam. Nowak \& Zhou (1990) suggest that system failure occurs when a group of adjacent girders fails. Tabsh \& Nowak (1991) postulated that 'several' girders must reach their ultimate strengths before the structure collapses in a series-parallel system. Liu \& Moses (1991) evaluated system reliability of bridge with damaged members. Moses (1997) generalized a system formulation in which the system fails either by reaching a maximum load level or else by attaining unserviceable large displacements. Ghosn \& Moses (1998) defined the ultimate capacity limit as the maximum possible truck load that can be applied on the structure before structure collapses defined as the formation of a collapse mechanism. A six-pre-stressed-concrete-girder bridge is evaluated for (i) the ultimate capacity of one girder reaching its maximum plastic rotation (equal to the 
maximum allowable rotation of $0.0402 \mathrm{rad}$ ) and (ii) the ultimate capacity of the mechanism formed with six overstressed girders whose ultimate capacity is six times the reserve capacity of one member. Moses (1982) proposed a general solution to find out multi-collapse mode. Estes \& Frangopol (1999) considered the failure of any three adjacent girders out of the simplified five-girder bridge model tantamount to system failure. Enright \& Frangopol (1999) analysed several system models for a five girder bridge.

A recent NCHRP Project (Roeder et al 2002) concluded that deformations that cause bridge damage are relative deflections between adjacent girder members, local rotations and deformations. One of the earliest deformation based failure definition for bridges was based on avoiding the undesirable structural effects and undesirable psychological reaction (ASCE 1958) and a limit of span/800 for steel bridges (simple as well as continuous spans) under live load plus impact was suggested. Based on 'the limit of visual observation', Galambos (1993) proposed using a maximum permanent or residual deflection equal to span/300 as serviceability limit state in bridge inelastic rating. As stated above, Ghosn \& Moses (1998) considered span/100 as 'dangerously high levels' of deformation.

It is thus clear that a crisp definition of system failure arises naturally only in a few idealized instances, e.g. in terms of the formation of a complete mechanism in a framed structure composed of elastic-perfectly plastic material, as assumed by Moses et al (1993b) and Eamon \& Nowak (2004). In a realistic situation, however, identification of system failure is often beset with uncertainty, and instead of forcing a precise definition of a subjective quantity, it may be more prudent to adopt a metric of failure that can incorporate the associated uncertainties, perhaps in the form of a random variable or a random process. To the knowledge of the authors, uncertainties in failure criteria have not received an adequate attention in the available literature on structural systems reliability.

\subsection{Proposed system failure criteria}

In light of the above discussion, we propose the following criterion for the system failure of girder bridges: Failure of a random number of $\bar{R}$ elements (an element here denotes a critical cross section of a girder) out of the total $n$ elements amounts to failure of the bridge structural system. In other words, the bridge system is conditionally an $R$ out of $n$ active parallel system with load sharing and load redistribution where $R=n-\bar{R}+1$. This system failure criterion does not necessarily correspond to total structural collapse, but to a substantial disruption in traffic owing to excessive local deformation of the roadway or unacceptable bridge dynamic behaviour.

Importantly in this context, $\bar{R}$ is a random variable. Randomness in $\bar{R}$ arises from (i) a manyto-many map between the numbers of failed elements and failed girders (in the case of indeterminate structures) and hence the maximum deflection, and (ii) subjectivity in the definition of the failure deflection criterion. The statistics of $\bar{R}$ may be determined from a small set of detailed numerical analyses. We found it preferable to define system failure in terms of a random number of failed elements $(\bar{R})$ : analysing a structure repeatedly up to states of large deformation is likely to be more expensive than searching for plastic hinges formed in the structure using one of several algorithms mentioned in the previous section. This approach also decouples system analyses from failure criteria and can potentially save significant computational efforts.

\subsection{Formulation of system reliability}

Assume that exactly $\bar{r}$ elements have failed. This way, the structural analysis and uncertain failure criteria can be decoupled as discussed above. In the $j^{\text {th }}$ load case, the $i^{\text {th }}$ cut set can 
be expressed as:

$$
C_{i}^{(j)}=\bigcap_{l \in L_{i}^{(j)}} F_{l}^{(j)}
$$

where $F_{l}^{(j)}$ is the failure of element $l$ and $L_{i}^{(j)}(\bar{r})$ is the set of all element indexes that constitute the $i^{\text {th }}$ cut set and is a function of $\bar{r}$ above. If the elements in a given cut set fail one at a time, a total of $n_{i}^{(j)}$ ! ordered sequences exist for cut set $i$ where $n_{i}^{(j)}=\left|L_{i}^{(j)}\right|$ is the size of the $i^{\text {th }}$ cut set. The way they are defined, these sequences are mutually exclusive, and as stated above, these are generally not equally likely. A major task in system reliability analysis is to identify the probabilistically dominant sequences.

Considering all sequences in cut set $i$ under load case $j$, we can write:

$$
C_{i}^{(j)}=\bigcup_{k=1}^{n_{i}^{(j)} !} C_{i, k}^{(j)}
$$

where the $k^{\text {th }}$ ordered sequence corresponding to cut set $i$ in load case $j$ is

$$
C_{i, k}^{(j)}=F_{l_{1}, 1}^{(j)} F_{l_{2}, 2}^{(j)} \ldots F_{l_{n_{i}^{(j)}}^{(j)}, n_{i}^{(j)}}^{(j)}, \quad\left\{l_{1}, l_{2}, \ldots, l_{n_{i}^{(j)}}\right\}=\mathrm{P}_{k}\left(L_{i}^{(j)}\right) .
$$

$F_{l_{p}, m}^{(j)}$ denotes the case when failure of element $l_{p}$ is the $m^{\text {th }}$ failure in the sequence under load case $j$, and $\mathrm{P}_{k}\left(L_{i}^{(j)}\right)$ denotes $k^{\text {th }}$ permutation (and hence the $k^{\text {th }}$ sequence) of the set of indexes $L_{i}^{(j)}$. The index $k$ runs up to $n_{i}^{(j)}$ ! which is total number of permutation of such sequences for cut set $i\left(n_{i}^{(j)}=\left|L_{i}^{(j)}\right|\right.$ being the size of the $i^{\text {th }}$ cut set $)$.

The system failure event can now be written as:

$$
F_{\mathrm{sys}}=\bigcup_{j=1}^{n_{L}} F_{\mathrm{sys}}^{(j)}=\bigcup_{j=1}^{n_{L}} \bigcup_{i=1}^{n_{j}} \bigcup_{k=1}^{n_{i}^{(j)} !} C_{i, k}^{(j)} .
$$

The computational demand in estimating system failure probability in case of large structures is obvious from (8). Simplifications are possible for girder bridge systems, as will be shown in section 4.

Finally, as discussed above, if the uncertainty in defining system failure criteria cannot be neglected, the system failure probability can be computed as:

$$
P\left[F_{\mathrm{sys}}\right]=\sum_{\operatorname{all} \bar{r}} P\left[\bigcup_{j=1}^{n_{L}} \bigcup_{i=1}^{n_{j}} \bigcup_{k=1}^{\left(n_{i}^{(j)}(\bar{r})\right) !} C_{i, k}^{(j)}(\bar{r}) \mid \bar{R}=\bar{r}\right] P[\bar{R}=\bar{r}]
$$

where $P[\bar{R}=\bar{r}]$ may be interpreted as the fraction of times system failure is caused by the failure of exactly $\bar{r}$ components such that $\sum_{\text {all } \bar{r}} P[\bar{R}=\bar{r}]=1$.

\section{Numerical example}

\subsection{The load and structural models}

In this section, we apply the methodology as in section 3 to an elementary example involving a simply-supported 5-girder steel bridge. The dimensions of the bridge are illustrated in 


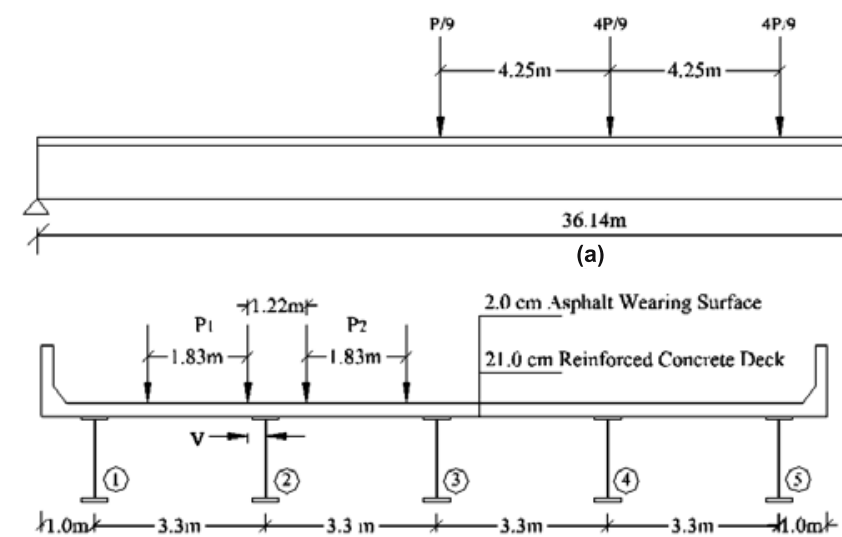

(b)

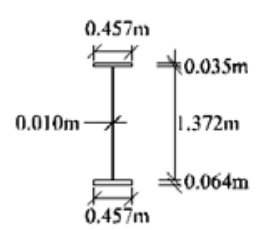

(c)

Figure 1. (a) Longitudinal location of truck on bridge, (b) deck cross-section, (c) girder cross-section.

figure 1 , girders are numbered from 1 to 5 . Only flexural failure is considered, and since the bridge is simply supported, the number of components, $n$, (defined as the number of critical cross sections) from a reliability point of view also is five. Flexural failure of a simply supported girder is defined as complete plastification of its critical cross-section. The girders are nominally identical and no aging effect is considered. Composite action is neglected, no source secondary stiffness is considered either. Element failure is irreversible. The material behaviour is elastic perfectly plastic, hence once a plastic hinge is formed, a girder does not carry any additional load. Live load is applied incrementally and proportionately until either global instability occurs (i.e. all 5 girders yield) or the load reaches its final value without causing global instability. The spread of plasticity through a member after failure of a section is neglected.

The incremental loading analysis is implemented in ANSYS following the work of Karamchandani and Cornell (Karamchandani 1990; Karamchandani \& Cornell 1992). Details of the finite element modelling for the bridge may be found in Zhong (2005). The load factors at the $k^{\text {th }}$ stage (i.e. after $k$ components have failed) for the surviving components are:

$$
\gamma_{i_{k}}^{(k)}=\frac{R_{i_{k}}-\sum_{q=0}^{k-1}\left[\gamma_{i_{q}}^{(q)}\left(\prod_{p=1}^{q}\left(1-\gamma_{i_{p-1}}^{(p-1)}\right) \cdot \sum_{j=1}^{m}\left(\alpha_{i_{k} j}^{(q)} X_{j}\right)\right)\right]}{\prod_{p=0}^{k-1}\left(1-\gamma_{i_{p}}^{(p)}\right) \cdot \sum_{j=1}^{m}\left(\alpha_{i_{k} j}^{(k)} X_{j}\right)}
$$

where $R_{i_{k}}$ is the resistance of component $i_{k} ; X_{j}$ is the $j^{\text {th }}$ of $m$ loads; and $\alpha_{i_{k} j}^{(k)}$ is the influence coefficient on component $i_{k}$ due to load $X_{j}$ at the $k^{\text {th }}$ stage. The analysis moves to the $(k+1)^{\text {th }}$ stage if any of the $\gamma_{i_{k}}^{(k)}$, s is less than one, in which case the component with the smallest $\gamma_{i_{k}}^{(k)}$ is considered to have failed. Otherwise, the number and id's of the components failed are stored, and the final deflection is computed as:

$$
U_{\varphi}^{k}=\sum_{q=0}^{k}\left[\gamma_{i_{q}}^{(q)}\left(\prod_{p=1}^{q}\left(1-\gamma_{i_{p-1}}^{(p-1)}\right) \cdot \sum_{j=1}^{m}\left(\beta_{\varphi j}^{(q)} X_{j}\right)\right)\right]+\prod_{p=0}^{k}\left(1-\gamma_{i_{p}}^{(p)}\right) \cdot \sum_{j=1}^{m}\left(\beta_{\varphi j}^{(k)} X_{j}\right) .
$$


Table 1. Dead load and resistance statistics.

\begin{tabular}{|c|c|c|c|}
\hline $\begin{array}{l}\text { Random } \\
\text { variable }\end{array}$ & $\begin{array}{l}\text { Resistance moment } \\
\text { capacity }\end{array}$ & $\begin{array}{c}\text { Concrete (plus } \\
\text { asphalt) dead load }\end{array}$ & $\begin{array}{l}\text { Concrete (plus } \\
\text { Steel dead load }\end{array}$ \\
\hline Nominal value & $11860(\mathrm{kNm})$ & $5.82\left(\mathrm{kN} / \mathrm{m}^{2}\right)$ & $4.54(\mathrm{kN} / \mathrm{m})$ \\
\hline Probability Distribution & Lognormal & Normal & Normal \\
\hline Bias & $1 \cdot 11$ & 1.05 & 1.03 \\
\hline Mean & $13165(\mathrm{kNm})$ & $6 \cdot 11\left(\mathrm{kN} / \mathrm{m}^{2}\right)$ & $4.68(\mathrm{kN} / \mathrm{m})$ \\
\hline c.o.v. & $0 \cdot 12$ & $0 \cdot 10$ & 0.08 \\
\hline
\end{tabular}

The first part in (11) is the deflection accumulated at the time of the $k^{\text {th }}$ component failure, the second part is the additional deflection due to the redistribution of the remaining part of external loads at the end of the $k^{\text {th }}$ stage. $\beta_{\varphi j}^{(q)}$ is the deflection influence coefficient due to external load $X_{j}$ at the $q^{\text {th }}$ stage.

Two load cases, both due to live load, are considered:

(i) LC1 - a single truck with at least one wheel line directly on one girder.

(ii) LC2 - two trucks side by side moving in the same direction separated by a specified minimum distance with at least one wheel line out of the four directly on one girder.

The problem is treated as time-invariant. An example of time-dependent reliability of highway girder bridges in the presence of cumulative aging effects and random pulse load processes, and how such first passage problems may be tackled can be found in Bhattacharya et al (2008).

System failure and uncertainty in failure criteria are discussed next.

\subsection{Statistics of basic random variables}

The component design equation against dead and live loads in AASHTO LRFD Guide is:

$$
\varphi R_{n}=\gamma_{D} D_{n}+\gamma_{L} L_{n}
$$

where $R_{n}$ is the nominal value of resistance, $D_{n}$ is the nominal value of dead load effect and $L_{n}$ is nominal value of the live load effect. $\varphi, \gamma_{D}$ and $\gamma_{L}$ are the resistance factor, dead load factor and live load factor, respectively. For steel components in flexure, the factors are: $\varphi=1, \gamma_{D}=1.25$ and $\gamma_{L}=1.75$.

The five girder simply supported steel bridge has already been described in figure 1 . The dead load has three sources: self-weights of the in-situ concrete deck, the steel girder and the asphalt. The statistics are shown in table 1. The statistical properties of live load for the two load cases are shown in table 2 . These statistics are based on values commonly adopted in the literature, e.g. Nowak (1995).

The deck is considered non-composite for design purposes in this example. The nominal dead load moment is $4668 \mathrm{kNm}$; the girder distribution factor is found to be 0.707 causing a nominal live load moment of $3443 \mathrm{kNm}$. The required nominal resistance thus is $11860 \mathrm{kNm}$. The nominal yield strength is $50 \mathrm{ksi}$ producing a nominal resistance of $11065 \mathrm{kNm}$ without considering contribution from the concrete deck. In the following, however, we will continue to use the code specified value of $11860 \mathrm{kNm}$ for $R_{n}$.

Statistical dependence among the girder strengths is accounted for in this example. It is assumed that each girder has identical marginal statistics and is lognormally distributed, and 
Table 2. Live load statistics.

\begin{tabular}{lcc}
\hline & Load case 1 & Load case 2 \\
\hline Nominal truck & One HS-20 $(320 \mathrm{kN})$ & 2 HS-20 side by side $(320 \mathrm{kN}$ each) \\
Multiple presence factor & 1.0 & 0.85 \\
Impact factor & 1.33 & 1.33 \\
Probability Distribution & Gumbel & Gumbel (marginal and joint) \\
Bias & $2 \cdot 0$ & $2 \cdot 0$ \\
c.o.v. & $0 \cdot 18$ & $0 \cdot 18$ \\
\hline
\end{tabular}

they are jointly lognormal as well. The correlation coefficient between each pair $(i, j)$ is constant and equal to $\rho_{R}$. Three different values of $\rho_{R}$ have been tried later in the example: $0,0.5$ and 1.0 . The following form is adopted for the multivariate lognormal distribution for the vector $\mathbf{X}$ with correlation matrix $\rho$ :

$$
F_{\mathbf{x}}(\mathbf{x})=\boldsymbol{\Phi}(\mathbf{z} ; \hat{\boldsymbol{\rho}}), \text { where } z_{i}=\left(\ln x_{i}-\mu_{i}\right) / \sigma_{i},
$$

where $\boldsymbol{\Phi}$ is the multidimensional standard Gaussian distribution function, $\hat{\boldsymbol{\rho}}$ is the matrix of correlation coefficients for $\mathbf{z} ; \mu_{i}$ and $\sigma_{i}$ are respectively the mean and standard deviation of $\ln X_{i}$, and $\hat{\rho}_{i j}$ needs to be derived from $\rho_{i j}$, as shown in der Kiureghian \& Liu (1986).

Statistical dependence among truck weights in load Case 2 has also been considered. Truck weights are assumed to be marginally Gumbel; their joint distribution is assumed bivariate Gumbel. Three different values of $\rho_{L}$, the correlation coefficient between truck weights in Load Case 2, have been used: 0, 0.5 and 1.0. The following form of the bivariate Gumbel CDF has been used in this paper (Gumbel \& Mustafi 1967):

$$
F_{X, Y}(x, y)=\exp \left\{-\left[\left(-\ln F_{X}(x)\right)^{m}+\left(-\ln F_{Y}(y)\right)^{m}\right]^{1 / m}\right\}
$$

such that $X$ and $Y$ are each marginally Gumbel with CDFs $F_{X}$ and $F_{Y}$, respectively. The parameter $m=1 / \sqrt{1-\rho}$ where $\rho(0 \leq \rho<1)$ is the correlation coefficient between $X$ and $Y$.

\subsection{Description of system failure}

In this example, we define failure of the bridge as the maximum deflection in the deck, $\Delta_{\max }$, exceeding the limit $\Delta_{0}$. We emphasize that $\Delta_{0}$ is a random variable, and based on the discussion in section 3, assume that it is Normally distributed with a mean $l / 100$ (where $l$ is the span length) and a coefficient of variation (c.o.v.) of $20 \%$. Note that this deflection limit is more severe than loss of serviceability and amounts to permanent inelastic deformation; it also includes the case of flexural failure of all 5 girders. The uncertainty in the failure criteria (cf. (9)) in terms of the probability function of $\bar{R}$, the number of girders involved in system failure, may be estimated as:

$$
P[\bar{R}=\bar{r}] \approx \frac{\#(\text { failures with } \bar{R}=\bar{r})}{\#(\text { all failures })}=\frac{\#\left(N_{F}=\bar{r} \cap \Delta_{\max }>l / 100\right)}{\#\left(\Delta_{\max }>l / 100\right)},
$$

where $N_{F}$ denotes the number of failed elements.

We believe that an important factor contributing to the above uncertainty is the randomness in the lateral location (indicated by $v$ in figure $1 \mathrm{~b}$ ) of trucks on the bridge. This aspect has 


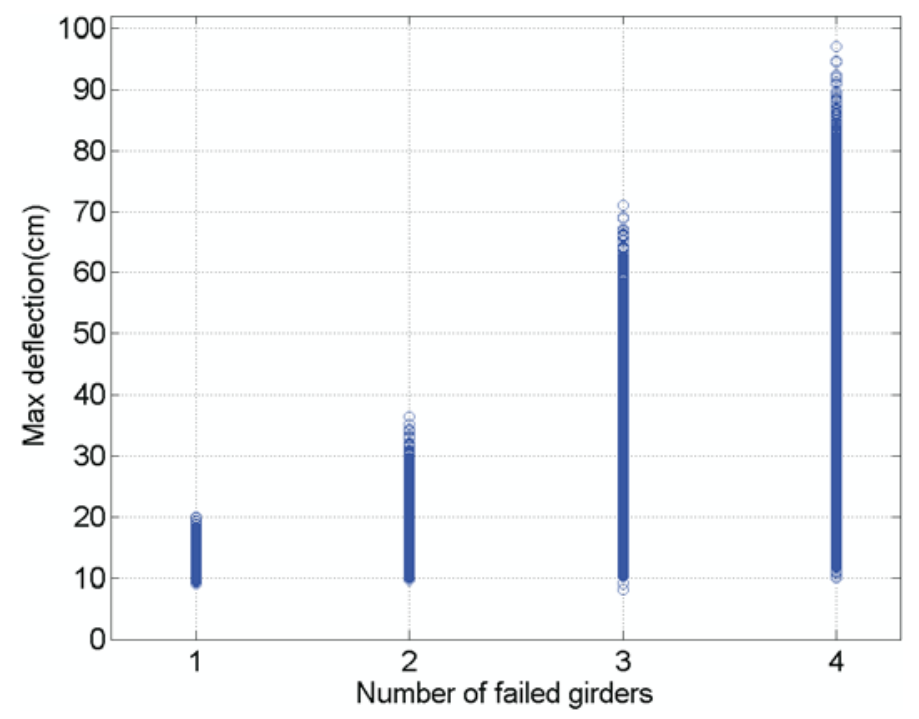

Figure 2. Maximum deflection of the bridge deck as a function of the number of girders failed in the 10 -factor experiment.

not been addressed adequately in existing work on bridge system reliability. To estimate the ratio on the right of (15), we designed an experiment involving 10 factors: strength of the five girders, two dead loads, two truck weights, and lateral location of a wheel line with respect to the girder. The following levels were chosen for the factors yielding 442,368 experimental configurations: $\mu, \mu-1.33 \sigma,-2.67 \sigma \mu-4 \sigma$ for the resistances; $\mu, \mu+2 \sigma, \mu+4 \sigma$ or the dead loads; $\mu, \mu+1.33 \sigma, \mu+2.67 \sigma, \mu+4 \sigma$ or the live loads; and $-2 \mathrm{ft}, 0 \mathrm{ft},+2 \mathrm{ft}$ for the lateral location, $v$. Since our objective was to focus on bridge failures, benign configurations (such as higher than mean resistances and lower than mean loads) were not considered in the experiment as they would not affect the results. Out of the 442,368 configurations, 41063 produced deflections above $l / 100$ of which 4911 corresponded to 5 girder failures. Figure 2 shows the distribution of maximum deflection as a function of number of girders failed; since the material behaviour is assumed elastic perfectly plastic, the deflection corresponding to 5 girder failure is indeterminate and has not been shown. Table 3 shows the estimated distribution of the failure criteria.

For $\bar{R}=2$, the system failure event (8) can be expressed as:

$$
F_{\mathrm{sys}}=\bigcup_{j=1}^{n_{L}} F_{\mathrm{sys}}^{(j)}=\bigcup_{j=1}^{n_{L}} \bigcup_{\substack{l, k=1 \\ l \neq k}}^{n} F_{l, 1}^{(j)} F_{k, 2}^{(j)},
$$

where $F_{l, 1}^{(j)}=$ element $l$ fails $1^{\text {st }}$ in load case $j$ and $F_{k, 2}^{(j)}=$ element $k$ fails $2^{\text {nd }}$ in load case $j$. Interchanging the order of unions, (16) can be rewritten as:

$$
F_{\mathrm{sys}}=\bigcup_{\substack{l, k=1 \\ l \neq k}}^{n}\left\{\bigcup_{j=1}^{n_{L}} F_{l, 1}^{(j)} F_{k, 2}^{(j)}\right\} \text {. }
$$


For all ordered pairs $(l, k)$ with $l \neq k$, the events $\bigcup_{j} F_{l, 1}^{(j)} F_{k, 2}^{(j)}$ are mutually exclusive. Hence, the system failure probability given $\bar{R}=2$ is

$$
P\left[F_{\text {sys }} \mid \bar{R}=2\right]=\sum_{\substack{l, k=1, \ldots, n \\ l \neq k}} P\left[\bigcup_{j} F_{l, 1}^{(j)} F_{k, 2}^{(j)}\right]
$$

We now take advantage of the simplification offered by the definition of the two load cases above. It is clear that in each load case, one wheel line is always directly over one girder. The loading geometry is thus defined relative to one of the girders. Denoting the transverse location of the said wheel line by $H$, it is clear that $H$ is a discrete random variable with $n$ possible values. The system failure probability given $\bar{R}=2$ then becomes:

$$
P\left[F_{\text {sys }} \mid \bar{R}=2\right]=\sum_{i=1}^{n} \sum_{\substack{l, k=1,2, \ldots, n \\ l \neq k}} P\left[\bigcup_{j} F_{l, 1}^{(j)} F_{k, 2}^{(j)} \mid H=h_{i}\right] P_{H}\left(h_{i}\right) .
$$

If we now make the reasonable assumption that the girder directly under one of the wheel lines is the first to fail, the number of terms in the summation can be significantly reduced:

$$
\begin{aligned}
P\left[F_{\mathrm{sys}} \mid \bar{R}=2\right] & =\sum_{i=1}^{n} \sum_{\substack{l, k=1,2, \ldots, n \\
l \neq k}} P\left[\bigcup_{j} F_{l, 1}^{(j)} F_{k, 2}^{(j)} \mid H=h_{i}\right] I(|l-k|=1) I(l=i) P_{H}\left(h_{i}\right) \\
& =\sum_{i=1}^{n} P\left[\bigcup_{j}\left(F_{i, 1}^{(j)} F_{i+1,2}^{(j)} \cup F_{i, 1}^{(j)} F_{i-1,2}^{(j)}\right) \mid H=h_{i}\right] P_{H}\left(h_{i}\right)
\end{aligned}
$$

in which $I$ denotes the indicator function. We further assume that $P_{H}\left(h_{1}\right)=P_{H}\left(h_{n}\right)=0$ (i.e. the edge girders do not have any wheel line directly on them) which allows us to simplify (20) as:

$$
P\left[F_{\mathrm{sys}} \mid \bar{R}=2\right]=\sum_{i=1}^{n} p_{2}^{(\mathrm{all} j)} P_{H}\left(h_{i}\right)=p_{2}^{(\text {all } j)}
$$

since $\sum_{i=1}^{n} P_{H}\left(h_{i}\right)=1$. The term $p_{2}^{\text {(all } j)}$ denotes the failure probability of two adjacent girders in all load cases when one wheel line is directly one of the said girders. Since $p_{2}^{(\text {all } j)}$ does not depend on $i$, the computation of system failure probability does not require looking at all failure sequences, but only at one representative case. Note that this simplification does not require $P_{H}\left(h_{i}\right)$ to be same for all $i$. Of course, if the girders are not equally sized or if they have aged differentially, then this simplification is no longer possible. The system failure probability given $\bar{R}=3,4$ can be similarly derived. 
Table 3. Distribution of number of failed girders from the 10-factor experiments.

\begin{tabular}{lccccc}
\hline \# failed girders & 1 & 2 & 3 & 4 & 5 \\
\hline \# cases above $l / 100$ & 0 & 44 & 11384 & 24724 & 4911 \\
$P[\bar{R}=\bar{r}]$ & 0 & 0.0084 & $0 \cdot 2540$ & 0.6194 & $0 \cdot 1181$ \\
\hline
\end{tabular}

\subsection{Results and discussion}

For each value $\bar{r}$ of $\bar{R}$, the failure probability of the system is calculated using importance sampling:

$p_{\bar{r}}^{(\text {all } j)}=\int_{-\infty}^{\infty} I_{D(\bar{r})}(\mathbf{x}) f_{\mathbf{X}}(\mathbf{x}) d \mathbf{x}=\int_{-\infty}^{\infty} I_{D(\bar{r})}(\mathbf{x}) \frac{f_{\mathbf{X}}(\mathbf{x})}{f_{\mathbf{V}}(\mathbf{x})} f_{\mathbf{V}}(\mathbf{x}) d \mathbf{x}=E_{\mathbf{V}}\left[I_{D(\bar{r})}(\mathbf{x}) \frac{f_{\mathbf{X}}(\mathbf{x})}{f_{\mathbf{V}}(\mathbf{x})}\right]$,

where $D(\bar{r})$ is the failure domain given $\bar{R}=\bar{r}, \mathbf{X}$ is the vector of basic variables, $I$ is the indicator function, $f_{\mathbf{V}}(\mathbf{x})$ is the importance sampling density function and $f_{X}(\mathbf{x})$ is the density function of the basic variables. $E_{V}$ is the expectation calculated with respect to $f_{\mathbf{V}}(\mathbf{x})$. 3 values are chosen for each of the correlation coefficients as mentioned above, giving rise to 9 cases. The importance sampling density is chosen to be of the same type as the original density, but with shifted means. 30,000 simulations are carried out in each case; stability of the estimates is investigated thoroughly in each case.

The unconditional failure probability is computed in the fashion of (9) combining the results from tables 3 and 4 :

$$
P\left[F_{\mathrm{sys}}\right]=\sum_{i=1}^{n} p_{\bar{r}}^{(\text {all } j)} p_{\bar{R}}(\bar{r}),
$$

which is shown in table 5. It is clear that system reliability goes down with increasing correlation in either group of random variables (truck loads and girder strengths). There is a large spread $\left(7 \times 10^{-6} \sim 2 \times 10^{-11}\right)$ in the failure probabilities for the nine combinations of load

Table 4. Conditional bridge system failure probability for various combinations of strength and load dependence.

\begin{tabular}{cccccc}
\hline$\rho_{R}$ & $\rho_{L}$ & $p_{2}^{(\text {all } j)}$ & $p_{3}^{(\text {all } j)}$ & $p_{4}^{\text {(all } j)}$ & $p_{5}^{\text {(all } j)}$ \\
\hline 0.0 & 0.0 & $4.245 \times 10^{-11}$ & $5.469 \times 10^{-11}$ & $7.654 \times 10^{-15}$ & $3.884 \times 10^{-27}$ \\
& 0.5 & $1.952 \times 10^{-10}$ & $5.287 \times 10^{-10}$ & $2.379 \times 10^{-12}$ & $2.572 \times 10^{-25}$ \\
& 1.0 & $2.076 \times 10^{-10}$ & $6.543 \times 10^{-10}$ & $2.369 \times 10^{-12}$ & $2.006 \times 10^{-20}$ \\
0.5 & $0 \cdot 0$ & $4.371 \times 10^{-6}$ & $9.614 \times 10^{-8}$ & $4.580 \times 10^{-9}$ & $7.561 \times 10^{-17}$ \\
& 0.5 & $6.843 \times 10^{-6}$ & $2.514 \times 10^{-6}$ & $1.283 \times 10^{-7}$ & $4.084 \times 10^{-15}$ \\
& 1.0 & $1.191 \times 10^{-5}$ & $2.613 \times 10^{-6}$ & $1.291 \times 10^{-7}$ & $1.636 \times 10^{-14}$ \\
1.0 & $0 \cdot 0$ & $1.355 \times 10^{-5}$ & $1.709 \times 10^{-6}$ & $2.410 \times 10^{-7}$ & $5.345 \times 10^{-13}$ \\
& 0.5 & $5.928 \times 10^{-5}$ & $6.120 \times 10^{-6}$ & $2.022 \times 10^{-6}$ & $2.289 \times 10^{-12}$ \\
& 1.0 & $9.209 \times 10^{-5}$ & $1.710 \times 10^{-5}$ & $2.355 \times 10^{-6}$ & $4.226 \times 10^{-12}$ \\
\hline
\end{tabular}


Table 5. Bridge system failure probability for various combinations of strength and load dependence.

\begin{tabular}{ccccc}
\hline & & \multicolumn{3}{c}{$\rho_{L}$} \\
\cline { 3 - 5 } & & 0.0 & 0.5 & 1.0 \\
\hline$\rho_{R}$ & 0.0 & $1.909 \times 10^{-11}$ & $1.395 \times 10^{-10}$ & $2.202 \times 10^{-10}$ \\
& 0.5 & $6.424 \times 10^{-8}$ & $7.776 \times 10^{-7}$ & $8.678 \times 10^{-7}$ \\
& 1.0 & $7.000 \times 10^{-7}$ & $3.303 \times 10^{-6}$ & $6.593 \times 10^{-6}$ \\
\hline
\end{tabular}

and strength correlation coefficients considered in the analysis. These values compare well with values obtained for similar structures, e.g. $\beta_{\text {sys }}>8$ (i.e. $P_{f}<6 \times 10^{-16}$ ) for a six girder system and $\beta_{\text {sys }}=5.0$ (i.e. $P_{f}=2.9 \times 10^{-7}$ ) for a four girder system as reported in Moses et al (1993a). Further, the target system reliability index of 5.5 (i.e. target system $P_{f}=1.8 \times 10^{-8}$ ) for highway bridges in flexure as recommended by Nowak et al (1997) is also about the middle of the above range.

We now look at the effect of the system factor, $\varphi_{s}$, commonly used to modify the component design equation:

$$
\varphi_{s} \varphi R_{n}=\gamma_{D} D_{n}+\gamma_{L} L_{n}
$$

Figure 3 shows the effect of the system factor on the bridge system reliability index, $\beta_{s}=$ $\Phi^{-1}\left(1-P\left[F_{\text {sys }}\right]\right)$. First of all, it is clear that correlation among girder strengths has a

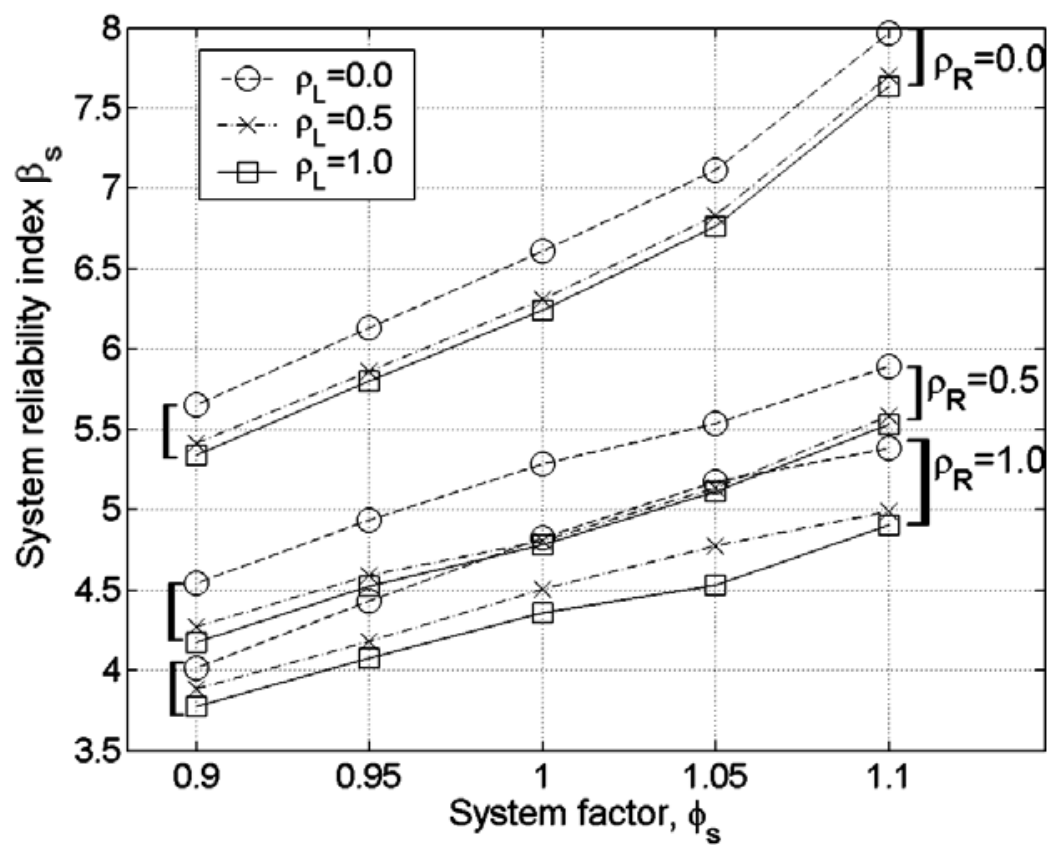

Figure 3. System reliability as a function of system factor for various level of correlation among girder strengths $\left(\rho_{R}\right)$ and truck weights $\left(\rho_{L}\right)$. 
significant, and detrimental, effect on the system reliability. Dependence on truck weights in multiple presence case also has a detrimental effect, although not as pronounced. Importantly, figure 3 shows the role of the system factor in affecting the bridge system reliability in this example: a mere change of 0.05 in $\varphi_{s}$ results in about one unit change in $\beta_{s}$ regardless of the correlation among the strength or load random variables. Similar figures may be developed for bridges of various configurations to aid the design process in achieving a desired system reliability.

\section{Conclusions}

This paper presented a formulation of structural system reliability in the presence of uncertainty in the definition of system failure. The formulation was geared toward failure of framed structures composed of elastic perfectly plastic materials, although it can easily be generalized. The decoupling of system analyses and system failure criteria can lead to savings in computational efforts. A detailed numerical example involving a steel highway girder bridge was presented. Uncertainty in system failure criteria was estimated, and structural system reliabilities for various combination of girder strength and truck load correlation were presented. The system factor to modify the component-level design equation to achieve a target system reliability was also illustrated.

\section{References}

AASHTO 1994 LRFD Highway Bridge Design Specifications. Washington, D.C., American Assoc. of State Highway and Transportation Officials

ASCE 1958 Deflection limitations of bridges, progress report of the Committee on Deflection Limitations of Bridges of the Structural Division. J. Structural Division, ASCE 84 (ST3)

Bennet R M, Ang A H-S 1986 Formulation of structural system reliability. J. Eng. Mech. ASCE 112(11): 1135-1151

Bhattacharya B, Basu R, Ma K-t 2001 Developing target reliability for novel structures: The case of the Mobile Offshore Base. Marine Structures 14(12): 37-58

Bhattacharya B, Li D, Chajes M J 2008 Bridge rating using in-service data in the presence of strength deterioration and correlation in load process. Structure and Infrastructure Engineering, Taylor and Francis 4(3): 237-249

Bjerager P, Karamchandani A, Cornell C A 1987 Failure tree analysis in structural system reliability. ICASP 5, Vancouver, Canada

Breitung K 1984 Asymptotic approximations for multinormal integrals. J. Eng. Mech. ASCE 110(3): 357-366

Cho H-N, Ang A H-S 1989 Reliability assessment and reliability-based rating of existing road bridges. 5th International Conference on Structural Safety and Reliability (ICOSSAR89), New York, ASCE

Cornell C A 1967 Bounds on the reliability of structural systems. J. Structural Division, ASCE ST1: $171-200$

Corotis R B, A M Nafday 1989 Structural system reliability using linear programming and simulation. J. Structural Eng. ASCE 115(10): 2435-2447

der Kiureghian A, Liu P-L 1986 Structural reliability under incomplete probability information. $J$. Eng. Mech. ASCE 112(1): 85-104

Ditlevsen O 1979 Narrow reliability bounds for structural systems. J. Structural Mech. 7(4): 453-472

Eamon C D, Nowak A S 2004 Effect of secondary elements on bridge structural system reliability considering moment capacity. Structural Safety 26(1): 29-47 
Enright M P, Frangopol D M 1999 Reliability-based condition assessment of deteriorating concrete bridges considering load redistribution. Structural Safety 21(7): 159-195

Estes A, Frangopol D M 1999 Repair optimization of highway bridges using system reliability approach. J. Structural Eng. ASCE 125(7): 766-775

Frangopol D M, Lin K-Y, Estes A 1997 Life-cycle cost design of deteriorating structures. J. Structural Eng. ASCE 123(10): 1390-1401

Galambos J 1993 The development of the mathematical theory of extremes in the past half century. Theory of probability and its applications 39(2): 234-248

Galambos T V 1990 System reliability and structural design. Structural Safety 7: 101-108

Ghosn M, Moses F 1998 Redundancy in highway bridge superstructures. Washington, DC, Transportation Research Board

Gorman M R 1981 Automatic generation of collapse mode equations. J. Structural Division, ASCE 107(ST7): 1350-1354

Gumbel E J, Mustafi C K 1967 Some analytical properties of bivariate extremal distributions. J. American Statistical Association 62(318): 569-588

Hohenbichler M, Rackwitz R 1983 First-order concepts in system reliability. Structural Safety 1: $177-188$

Karamchandani A 1987 Structural system reliability analysis methods. Reliability of Marine Structures Program. Stanford; CA; Department of Civil Engineering, Stanford University

Karamchandani A 1990 New methods in system reliability. Civil Engineering, Stanford University

Karamchandani A, Cornell C A 1992a An event-to-event strategy for nonlinear analysis of truss structures I. J. Structural Eng. ASCE 118(4): 895-909

Karamchandani A, Cornell C A 1992b Reliability analysis of truss structures with multistate elements II. J. Structural Eng. ASCE 118(4): 910-925

Liu Y, Moses F 1991 Bridge design with reserve and residual reliability constraints. Structural Safety 11: $29-42$

Moses F 1982 System reliability developments in structural engineering. Structural Safety 1: 3-13

Moses F 1997 Problems and prospects of reliability-based optimization. Engineering Structures 19(4): 293-301

Moses F, Ghosn M, Khedekar N C 1993a Development of redundancy factors for highway bridges. 10th International Bridge Conference, Pittsburgh

Moses F, Khedekar N C, Ghosn M 1993b System reliability of redundant structures using response function. International Conference on Structural Safety and Reliability, Innsbruck, Austria

NCHRP 1999 Calibration of LRFD bridge design code. Washington, D.C., Transportation Research Board, National Research Council

Nowak A S 1995 Calibration of LRFD bridge code. J. Structural Eng. ASCE 121(8): 1245-1251

Nowak A S, Szerszen M M, Park C H 1997 Target safety levels for bridges. 7th International Conference on Structural Safety and Reliability, Kyoto, Japan

Nowak A S, Zhou J 1990 System reliability models for bridges. Structural Safety 7(2-4): 247-254

Roeder C W, Barth K, Bergman A 2002 Improved Live Load Deflection Criteria for Steel Bridges, TRB

Shao S, Murotsu Y 1999 Approach to failure mode analysis of large structures. Probabilistic Eng. Mech. 14: 169-177

Tabsh S W, Nowak A S 1991 Reliability of highway girder bridges. J. Structural Eng. ASCE 117(8): 2372-2388

Tang L K, Melchers R E 1987 Improved approximation for multinormal integral. Structural Safety 4: 81-93

Thoft-Christensen P, Murotsu Y 1986 Application of Structural Systems Reliability Theory (Berlin: Springer-Verlag)

Tong Y L 1990 The Multivariate Normal Distribution (New York: Springer-Verlag)

Watwood V B 1979 Mechanism generation for limit analysis of frames. J. Structural Division, ASCE 109(ST1): 1-15 
Wen Y K 2001 Minimum lifecycle cost design under multiple hazards. Reliability Engineering and System Safety 73: 223-231

Zhong J 2005 System Reliability based Design of Steel Girder Bridges using Incremental Loading Method. Civil and Environmental Engineering. Newark, DE, University of Delaware

Zimmerman J J, Ellis J H, Corotis R B 1993 Stochastic optimization models for structural reliability analysis. J. Structural Eng. ASCE 119(1): 223-239 